\title{
Four-quark matter-a new era of spectroscopy
}

\author{
Zhiqing Liu
}

\begin{abstract}
In 1964, both Gell-Mann and Zweig proposed the famous Quark Model in particle physics, which tells us hadrons are built of three quarks (baryons) or quark anti-quark pair (mesons). However, the theory of strong interaction-QCD—allows the existing of hadrons beyond the conventional baryon and meson picture. These new hadron states are called exotic hadrons, and have been searched for over the past half century. In this review, I will introduce you the discovery of a new particle called $Z_{c}(3900)$, which is considered as the first convincing four-quark particle. The observation of four-quark matter gains great interest in particle physics, and triggered subsequent intensive study of exotic hadrons, which brings us to a new era of hadron spectroscopy and refreshes our knowledge about the hadronic matter in our universe.
\end{abstract}

\section{Introduction}

Particle physics basically study the fundamental structure, i.e., the elementary building blocks of matter in our universe-by far we know they are quarks, leptons, etc.-and also the fundamental force governing themthe strong, weak, electromagnetic, and gravity forces. Regarding the structure of matter, it is an old philosophy question, but also a very modern one. We know atoms have internal structure-they are built of nucleus and electrons; and nucleus are further built of protons and neutrons. Both protons and neutrons are still not elementary, they have internal structure and are built of quarks.

In the 1960s, there are lots of new particles observed both in cosmic rays and lab experiments. Classifying them in a proper way seems to be the only way out, since not all of them look elementary. Both Gell-Mann and Ne'eman developed a classification scheme called "Eightfold Way" - SU(3) flavor symmetry $[1,2]$, which arranges the particles into several multiplets and also predicts a missing $\Omega^{-}$baryon with a mass $1676 \mathrm{MeV} / \mathrm{c}^{2}$ in the baryon decuplet $[1,3]$. Shortly later in 1964, experiments at BNL

Correspondence: z.liu@sdu.edu.cn

Institute of Frontier and Interdisciplinary Science, Shandong University, Qingdao, Shandong Province 266237, China discovered it with a mass $1686 \pm 12 \mathrm{MeV} / c^{2}$ [4], which agrees with prediction well. The big success of the "Eightfold Way" in classifying particles leads to the birth of the Quark Model [5], which was proposed by Gell-Mann and Zweig independently and reveals the nature behind. In the Quark Model, quarks are introduced as the elementary particles. And hadrons, bound states of quarks via the strong interaction, are made of three quarks $q q q$ (called baryons) or quark anti-quark pairs $q \bar{q}$ (called mesons). The Quark Model also points out other quark combinations such as $q q \bar{q} q, q q q q \bar{q}$ could form hadrons, but not too much attention is caught just because the simple baryon and meson picture works so beautifully since then.

Nowadays, after 50 years, the Quark Model is still a powerful tool to describe hadrons. As an example, the LHCb experiment observed a new baryon $\Xi_{c c}^{++}$in 2017, with a mass $3621.24 \pm 0.65 \pm 0.31 \mathrm{MeV} / c^{2}$ and a life time $\tau=256_{-22}^{+24} \pm 14$ fs [6]. These properties agree well with the Quark Model prediction for a spin- $\frac{1}{2}$ baryon with a quark content $u c c$. Many other observations consistent with the Quark Model are also widely present by experiments recently [7].

The fundamental force to bind quark together is the strong interaction, and the theory to describe strong interaction is quantum chromodynamics (QCD). In the
Springer

(c) The Author(s). 2021 Open Access This article is licensed under a Creative Commons Attribution 4.0 International License, which permits use, sharing, adaptation, distribution and reproduction in any medium or format, as long as you give appropriate credit to the original author(s) and the source, provide a link to the Creative Commons licence, and indicate if changes were made. The images or other third party material in this article are included in the article's Creative Commons licence, unless indicated otherwise in a credit line to the material. If material is not included in the article's Creative Commons licence and your intended use is not permitted by statutory regulation or exceeds the permitted use, you will need to obtain permission directly from the copyright holder. To view a copy of this licence, visit http://creativecommons.org/licenses/by/4.0/. 
QCD framework, however, hadrons beyond the conventional baryons and mesons are allowed, such as the glueballs, multi-quark states, quark-gluon hybrid states, and hadronic molecules, etc. (see recent review articles [810]). All these new types of hadrons are usually referred to as exotic hadron states. A big challenge is that we do not know how to calculate quark binding-a low energy confinement problem in the non-perturbative regime of the QCD theory. Anyway, the existence of exotic hadrons has stimulated people's continuous interest to search for them in experiment.

In the early years, people try to search for exotic hadron states through light hadron production. The lattice QCD theory calculated the scalar glueball mass to be around $1.5-1.7 \mathrm{GeV} / c^{2}$ [11], and there are several candidates $f_{0}(1370), f_{0}(1500)$, and $f_{0}(1710)$ in experiment [7]. The question is, one cannot clearly identify them as a glueball due to serious mixing effect with conventional light mesons in this energy region. This situation improved a lot since 2003, when a heavy charmonium-like particle $X$ (3872) was observed in experiment [12]. Compared to light hadrons, heavy quarkonium states are isolated more clearly and also relatively easier to calculate, and thus, serve as an ideal place to test spectroscopy.

\section{Charmonium spectroscopy}

When talk about charmonium, we usually would like to make an analogy to the positronium system. Positronium is a bound state of electron and positron by pure Coulomb force, which is understood well and calculated precisely by the quantum electrodynamics theory (QED). The leading-order attractive Coulomb potential for the positronium system is $V_{e^{+}} e^{-}(r)=-\frac{\alpha}{r}$, where $\alpha$ is the finestructure constant and $r$ is the distance between electron and positron.

Charmonium is a bound state of charm $(c)$ and anticharm $(\bar{c})$ quarks via strong interaction. Due to much heavier mass of the charm quark $\left(m_{c} \sim 1.3 \mathrm{GeV} / c^{2}\right)$, the velocity of charm quark motion inside the charmonium is relatively slow. Thus, we can use a non-relativistic potential to describe the interaction between $c$ and $\bar{c}$. From the potential model [13] the leading-order potential can be written as

$$
V_{c \bar{c}}(r)=-\frac{4 \alpha_{s}}{3 r}+k r
$$

where $\alpha_{s}$ is the strong-coupling constant, $k$ is a linearconfinement parameter, and $r$ is the distance between $c$ and $\bar{c}$.

According to the asymptotic freedom feature of the strong interaction, the force between $c$ and $\bar{c}$ is relatively weak when $r \rightarrow 0$, and the potential is dominated by an attractive Coulomb potential, very similar to the positronium system. With the increasing of $r$, the confinement force becomes stronger, and the potential is dominated by a linear term. Figure 1 shows a typical potential of the charmonium system.

Once having the potential, we can solve the Schrödinger equation of the system and get the energy levels and the corresponding wave functions, like a hydrogen atom. Figure 2 shows the obtained charmonium spectrum [14], where we use the $n^{2 s+1} L_{J}$ notation to label an energy level, and $J^{P C}$ to label the spin, parity, and charged conjugation parity quantum numbers.

Below the open-charm $(D \bar{D}$ meson pair) mass threshold, there are in total 8 charmonium states predicted. And all of them have been well established in experiment [7]. The first candidate $J / \psi\left(1^{3} S_{1}\right)$ has been discovered in 1974 by groups leaded by Samuel Ting and B. Richter [15], which is awarded the Nobel Prize for the discovery of charm quark. The last candidate $h_{c}\left(1^{1} P_{1}\right)$ was observed by the CLEO-c experiment in 2005 [16]. Above the open-charm threshold, the charmonium spectrum is still in progress. Several vector states $\left(J^{P C}=1^{--}\right)$, such as the $\psi(3770)\left(1^{3} D_{1}\right)$, $\psi(4040)\left(3^{3} S_{1}\right), \psi(4160)\left(2^{3} D_{1}\right)$, and $\psi(4415)\left(4^{3} S_{1}\right)$, have been established quite some time ago [7], while a few more were only observed recently. In 2015, the BESIII experiment reported observation of the $X(3823)$ resonance with $6.2 \sigma$ significance in $e^{+} e^{-} \rightarrow \pi^{+} \pi^{-} X(3823) \rightarrow$ $\pi^{+} \pi^{-} \gamma \chi_{c 1}$ process [17]. The mass of $X(3823)$ is measured to be $3821.7 \pm 1.3 \pm 0.7 \mathrm{MeV} / c^{2}$, which agrees with the $1^{3} D_{2}$

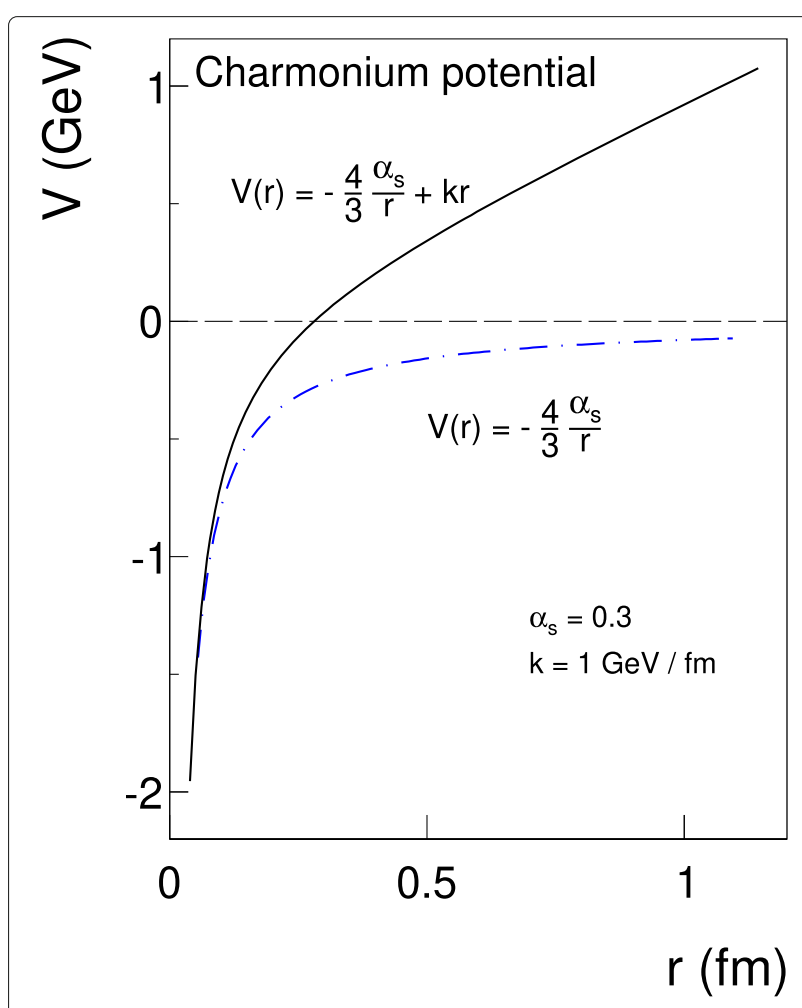

Fig. 1 The potential between $c$ and $\bar{c}$ for a charmonium system 


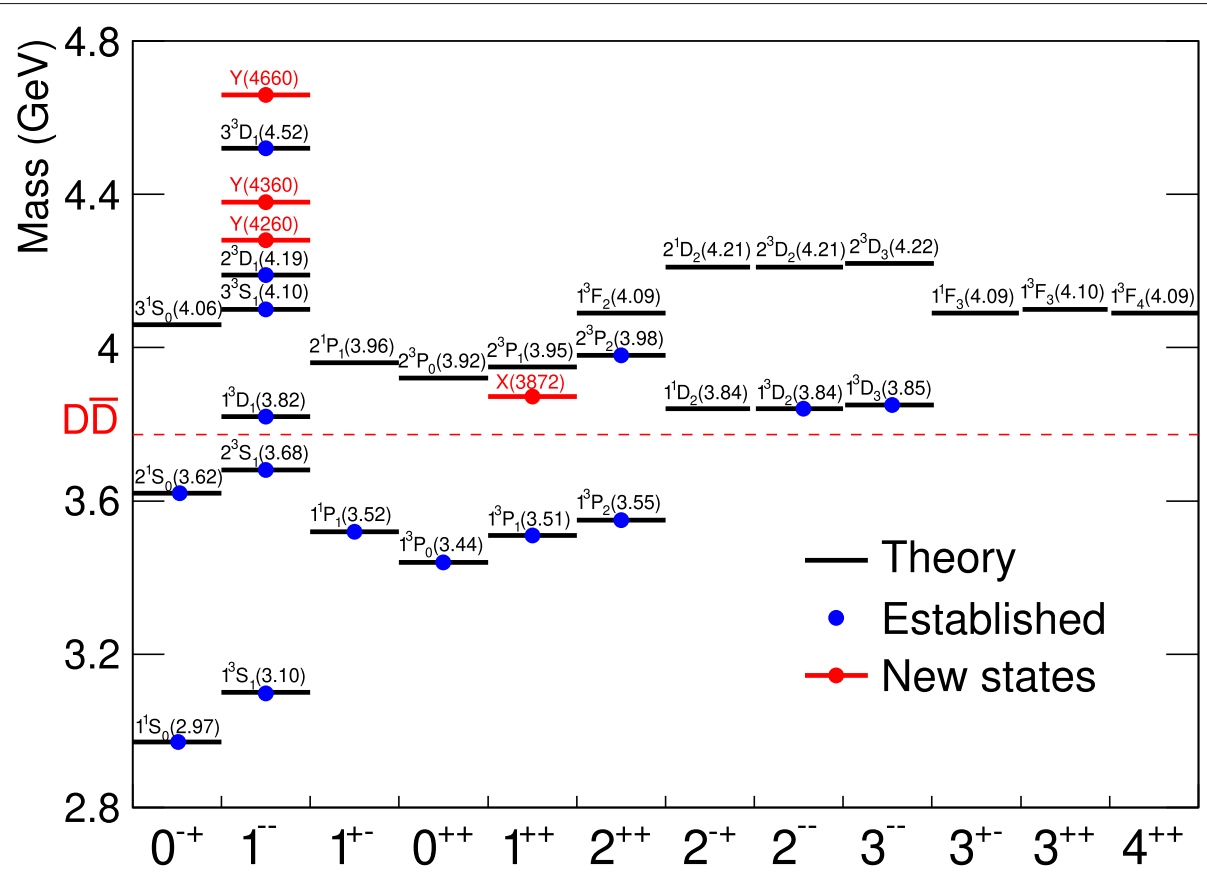

Fig. 2 The charmonium spectrum from potential model prediction

$\left(J^{P C}=2^{--}\right)$charmonium state very well. In 2019, the $\mathrm{LHCb}$ experiment also reported observation of the $1^{3} D_{3}$ $\left(J^{P C}=3^{--}\right)$state via $D \bar{D}$ final state [18].

If one looks at the full charmonium spectrum (c.f. Fig. 2), there are still many leaving "blank" energy levels in experiment above the $D \bar{D}$ mass threshold. When people are searching for these missing candidates, surprisingly, new particles such as the $X(3872)$ [12], $Y(4260)$ [19], $Y(4360)$, and $Y(4660)$ [20], etc. are observed in experiment. These new particles' mass locate in the charmonium energy region, yet cannot easily find a position in the predicted charmonium spectrum. People speculate they might be the missing exotic hadron states expected from QCD. Especially with the observation of the charged state $Z_{c}(3900)$ [21, 22], which carries electric charge, we are more confident some of these new particles observed in the charmonium energy region are not conventional hadrons, but exotic hadron state instead.

\section{Discovery of $Z_{c}(3900)$ at the BESIII and Belle experiments}

The BESIII experiment located in Beijing is a symmetric $e^{+} e^{-}$collision experiment, which runs at $e^{+} e^{-}$center-ofmass (c.m.) energy from 2.0 to $4.9 \mathrm{GeV}$. From December 2012 to January 2013, the Collaboration decided to run the machine at $\sqrt{s}=4.26 \mathrm{GeV}$, which corresponds to the $Y$ (4260) resonance [19] peak position. By that time, I was doing my $\mathrm{PhD}$ thesis at the BESIII experiment, and one of my topic is to study the $Y(4260) \rightarrow \pi^{+} \pi^{-} J / \psi$ decay.
To study the $Y(4260)$ particle, we need to take collision data first. Within 1 month time, the BESIII detector was able to accumulate $525 \mathrm{pb}^{-1}$ data at $\sqrt{s}=4.26 \mathrm{GeV}$, with a peak luminosity $\mathcal{L}_{\text {peak }}=5.3 \times 10^{32} \mathrm{~cm}^{-2} \mathrm{~s}^{-1}$. Figure 3 shows the luminosity history during BESIII data taking period. Compared with the CLEO-c experiment [23], BESIII's designed luminosity is one order of magnitude higher [24], and the data size is about 40 times larger at $\sqrt{s}=4.26 \mathrm{GeV}$.

During the data taking procedure, the Collaboration also carefully monitor everything, to ensure each subdetector system works well. A beam energy measurement system is used to monitor and measure the beam energy with a precision of $\pm 1.0 \mathrm{MeV}$ [25]. After data is recorded, we also calibrate every sub-detector carefully, including the multi-layer drift chamber, the time-of-fight detector, the electromagnetic calorimeter, and the muon counter, to obtain good-quality data. To verify our data quality, as an example, we measure the $D$ meson mass, and the difference to its world average value is $\delta[m(D)]=0.5 \pm$ $0.2 \mathrm{MeV} / c^{2}$; we also measured the $\psi(2 S)$ mass from our data, and the difference to its world average value is $\delta[m(\psi(2 S))]=0.2 \pm 0.1 \mathrm{MeV} / c^{2}$; here the errors are statistical. The mass resolution of $D$ meson measured from $D \rightarrow K \pi$ events is $6.0 \pm 0.1 \mathrm{MeV} / c^{2}$, and for $\psi(2 S) \rightarrow$ $\pi^{+} \pi^{-} J / \psi$ events is $2.0 \pm 0.1 \mathrm{MeV} / c^{2}$. All these measurements demonstrate our data quality is nice and available for further measurements.

The $Y(4260)$ particle is produced directly in $e^{+} e^{-}$ annihilation at BESIII and then subsequently decays to 


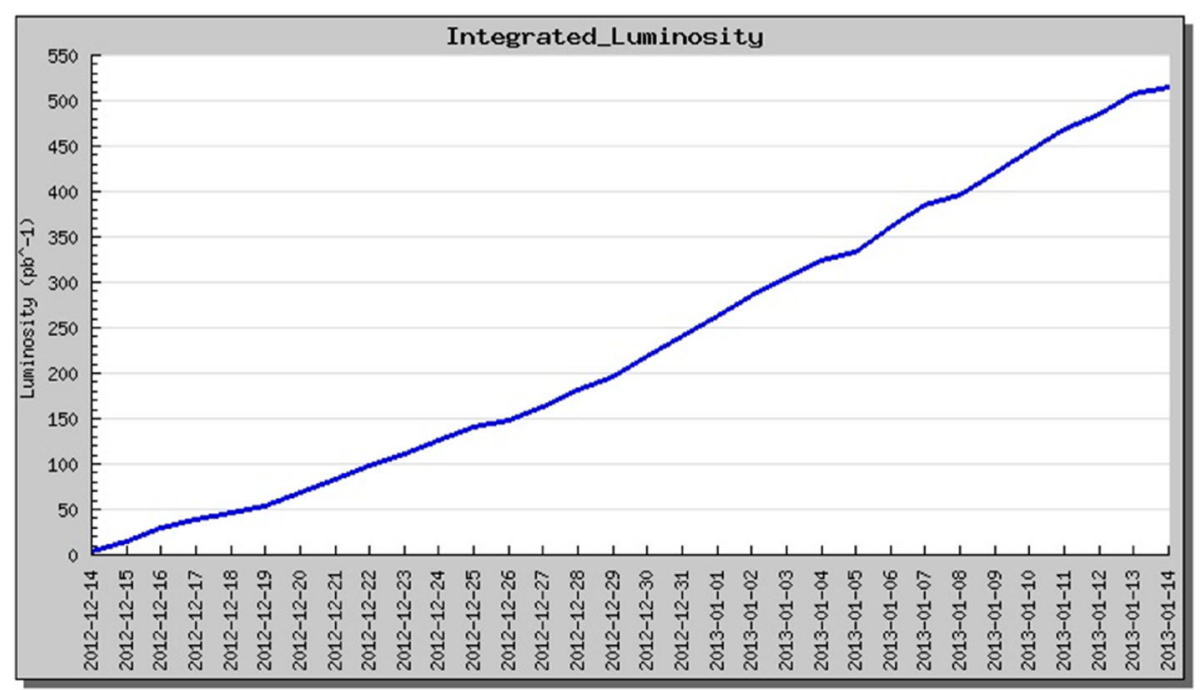

Fig. 3 The integrated luminosity collected by the BESIII detector from December 2012 to January 2013

$\pi^{+} \pi^{-} J / \psi$ with $J / \psi \rightarrow \ell^{+} \ell^{-}(\ell=\mu$ or $e)$. In a $1 \mathrm{~T}$ magnetic field within the BESIII detector, pions have relative low momentum, resulting in small radius of curvature for the tracks in the drift chamber (bend more "seriously"), while leptons have higher momentum, with much larger radius for the tracks (look more like "straight" lines). Figure 4 shows the event shape for a typical $Y(4260) \rightarrow$ $\pi^{+} \pi^{-} J / \psi \rightarrow \pi^{+} \pi^{-} \ell^{+} \ell^{-}$event inside the BESIII detector, which just simply looks like the greek letter " $\psi$ ", and now is widely used to name a charmonium state.

To effectively select $\pi^{+} \pi^{-} J / \psi$ signal events, we ask for four charged tracks in an event and reconstruct the $J / \psi$ particle by lepton pair (see details in Ref. [21]). Figure 5 shows the reconstructed lepton pair mass distribution, where significant $J / \psi$ signals are observed both in $\mu^{+} \mu^{-}$and $e^{+} e^{-}$channels. The noise and background level is well controlled and low. By fitting the mass distributions, we obtain about $1600 \pi^{+} \pi^{-} J / \psi$ signal events, with estimated background level $<10 \%$ (remaining background mainly come from $\pi^{+} \pi^{-} \pi^{+} \pi^{-}$ and $\mu^{+} \mu^{-} e^{+} e^{-}$events). To validate the analysis, we measure the $e^{+} e^{-} \rightarrow \pi^{+} \pi^{-} J / \psi$ production cross section to be $(62.9 \pm 1.9 \pm 3.7) \mathrm{pb}$ at $\sqrt{s}=4.26 \mathrm{GeV}$, which is consistent with previous measurements from the $B A B A R$ [19] and Belle [26] experiments, and with improved precision.

With the much larger data sample from BESIII, for the first time, we are able to search possible intermediate state in the $\pi^{+} \pi^{-} J / \psi$ process. Namely we study the Dalitz plot of the selected $\pi^{+} \pi^{-} J / \psi$ events and observe a band in the $M\left(\pi^{+} J / \psi\right)$ mass (or the $M\left(\pi^{-} J / \psi\right)$ mass) dimension. Figure 6 (left) shows the 1-dimensional projection of the $M\left(\pi^{ \pm} J / \psi\right)$ (by adding $M\left(\pi^{+} J / \psi\right)$ and $M\left(\pi^{-} J / \psi\right)$ distributions together) mass distribution, where a resonance structure near $3900 \mathrm{MeV} / \mathrm{c}^{2}$ is clearly seen. The BESIII experiment observed 307 signal event for this new resonance and measured its mass and width

$$
\begin{array}{r}
M=(3899.0 \pm 3.6 \pm 4.9) \mathrm{MeV} / c^{2}, \\
\Gamma=(46 \pm 10 \pm 20) \mathrm{MeV} .
\end{array}
$$

and name it as $Z_{c}$ (3900) for this discovery. The significance of the $Z_{c}(3900)$ resonance is $>8 \sigma$ in various systematic uncertainty tests. Possible $\pi^{+} \pi^{-}$resonances

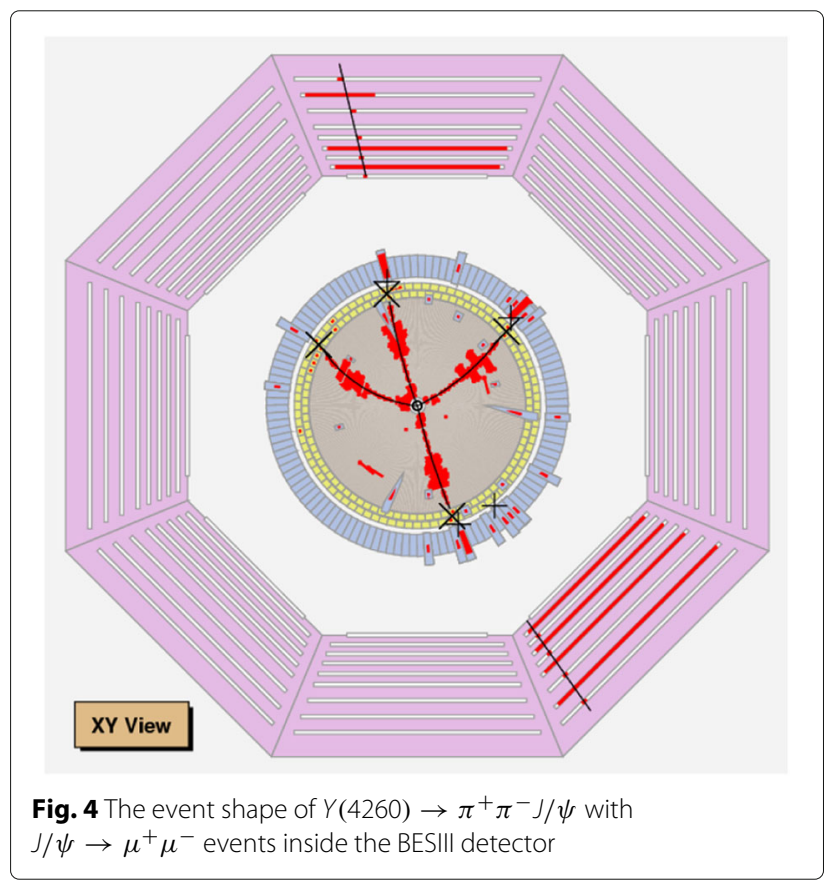



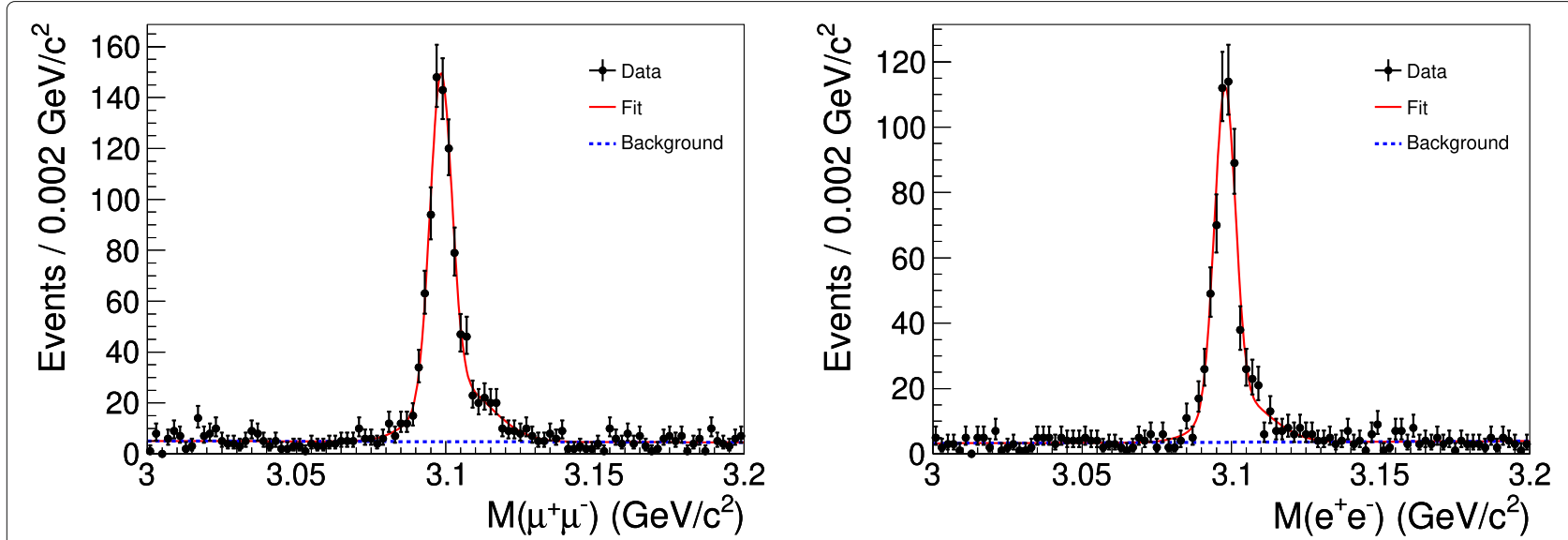

Fig. 5 The $M\left(\mu^{+} \mu^{-}\right)$(left) and $M\left(e^{+} e^{-}\right)$(right) invariant mass distributions from selected $Y(4260) \rightarrow \pi^{+} \pi^{-} J / \psi$ events at BESIII

are also carefully studied, and we find they are well modeled by scalar resonance (spin is 0) $f_{0}(500)$ and $f_{0}(980)$. Such kind of resonance with spin 0 will not produce enhancement in the $M\left(\pi^{ \pm} J / \psi\right)$ dimension due to reflection effect. A further amplitude analysis also determines the spin-parity of $Z_{c}(3900)$ to be $J^{P}=1^{+}$[27].

At the same time, the Belle experiment in Japan is also studying the $Y(4260) \rightarrow \pi^{+} \pi^{-} J / \psi$ events. Different from BESIII, the Belle experiment takes data at $e^{+} e^{-}$ c.m. energy near $10.6 \mathrm{GeV}$. Belle uses a so-called initialstate-radiation approach to produce the $Y(4260)$ particle, which also enable us to study the $Y(4260) \rightarrow \pi^{+} \pi^{-} J / \psi$ decay. Figure 6 (right) shows the $M\left(\pi^{ \pm} J / \psi\right)$ mass distribution obtained from $967 \mathrm{fb}^{-1}$ Belle data, and a resonance structure is also observed around $3900 \mathrm{MeV} / c^{2}$ [22]. Belle observed $159 Z_{c}(3900)$ signal events and measured its mass and width

$$
\begin{array}{r}
M=(3894.5 \pm 6.6 \pm 4.5) \mathrm{MeV} / c^{2}, \\
\Gamma=(63 \pm 24 \pm 26) \mathrm{MeV} .
\end{array}
$$

The significance of $Z_{c}(3900)$ estimated by Belle experiment is $>5.2 \sigma$ in various systematic uncertainty tests.

The discovery of $Z_{c}(3900)$ both by BESIII and Belle soon strikes world-wide attention in the particle physics community. The $Z_{c}(3900)$ resonance decays to a charged pion and a $J / \psi$. From its width, we know the lifetime of $Z_{c}(3900)$ is very short $\left(<10^{-23} \mathrm{~s}\right)$. The decay is through strong interaction, and $Z_{c}(3900)$ should contain $c$ and $\bar{c}$ inside. However, pure $c \bar{c}$ cannot explain $Z_{c}(3900)$ as it carries electric charge. As a result, we need as least an additional pair of quarks (such as $u \bar{d}$ ) to provide electric charge. Then, in this sense, the most natural interpretation for $Z_{c}(3900)$ is a four-quark particle. $Z_{c}(3900)$ is reported as "the first convincing four-quark particle" by
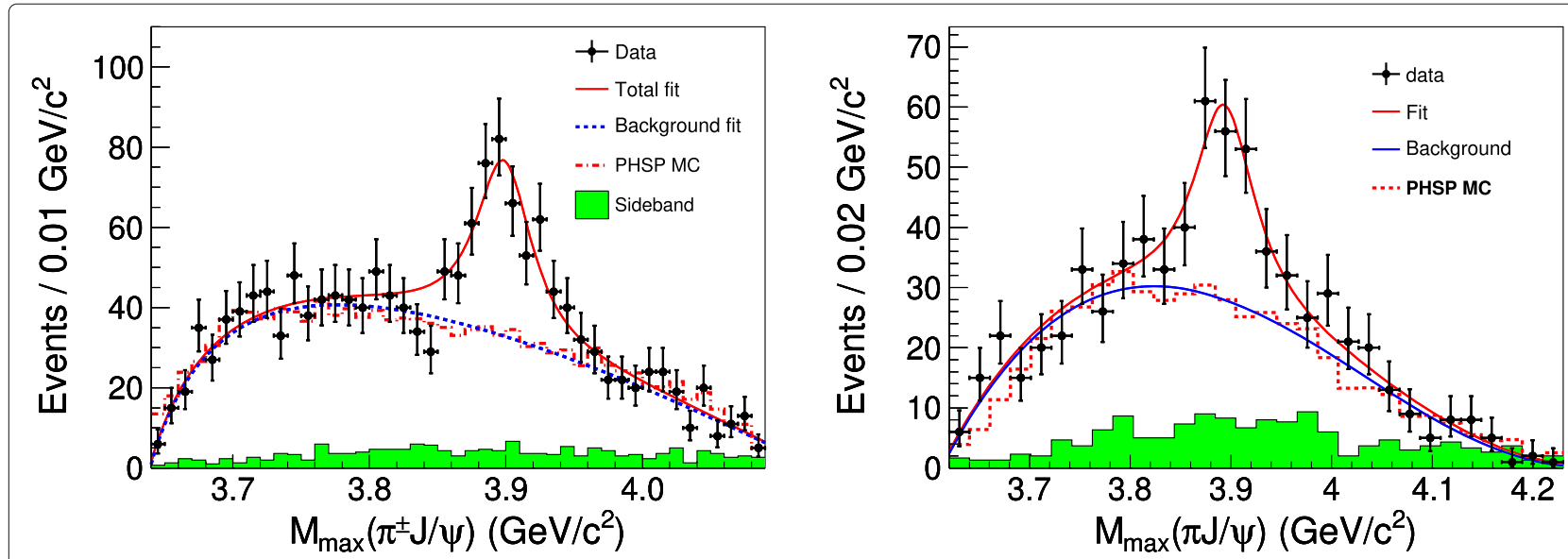

Fig. 6 The $M\left(\pi^{ \pm} J / \psi\right)$ mass distribution from BESIII (left) and Belle (right) experiments, where the $Z_{c}(3900)$ resonance is discovered 
Nature [28] and other social medias such as Wired Science [29], SPIEGEL Online [30], etc. The Physics journal from the American Physics Society in 2013 selected 11 standout stories as "Highlights of the Year", and Four-Quark Matter-the discovery of $Z_{c}(3900)$ is listed as top one event [31]. It is worth to mention, the $Z_{c}(3900)$ signal has been confirmed independently by the CLEO-c experiment 6 months later [32], and also the D0 experiment in 2019 [33].

\section{A new era of spectroscopy}

The discovery of four-quark particle brings a wave of new particle search in experiment. Until today, there are many more candidates - the so-called $X Y Z$ particles observed in experiment. These $X Y Z$ particles locate in the charmonium energy region, but cannot accommodate with the potential model-predicted charmonium spectrum easily, either for particle parameters (mass, width etc.) or for their production/decay behaviors. They are considered as very good candidates for exotic hadrons. Especially for the charged particles, they are more convincing exotic hadron states. Our traditional particle "zoo" is greatly enlarged, and we are entering into a new era of hadron spectroscopy. Here I list some most-discussed ones.

\subsection{The neutral $X(3872)$}

The $X$ (3872) resonance is the first $X Y Z$ particle observed in 2003 by the Belle experiment in $B \rightarrow K X(3872)$ process [12]. It has been later confirmed by at least 7 independent experiments [34-40], and thus, the signal is solid. There are a lots of studies about $X(3872)$ in the past decade. Its mass is measured precisely to be $3871.59 \pm 0.06 \pm$ $0.03 \pm 0.01 \mathrm{MeV} / c^{2}$ and width is $0.96_{-0.18}^{+0.19} \pm 0.21 \mathrm{MeV}$ by the $\mathrm{LHCb}$ experiment recently [41]. The spin-parity quantum numbers are measured to be $J^{P C}=1^{++}$[42]. If we look carefully at the potential model-predicted charmonium spectrum (c.f. Fig. 2), there is no position for the $X(3872)$ at all. The only possible $1^{++}$charmonium candidate is $\chi_{c 1}(2 P)$, which is expected to have a mass around $3960 \mathrm{MeV} / \mathrm{c}^{2}$ and width $>100 \mathrm{MeV}$. What is more, $X$ (3872) has big Iso-spin (an usually conserved quantum number in the strong interaction) violation effect, which is also quite different from a normal charmonium state. By searching for its Iso-spin partner, we know $X(3872)$ is a Iso-spin 0 state $[43,44]$. However, the decay rate of $X(3872) \rightarrow \rho J / \psi[44,45]$ and $\pi^{0} \chi_{c 1}[46]\left(\rho\right.$ and $\pi^{0}$ is Iso-spin one) are anomalously large, which is an order of magnitude higher than a typical charmonium state, such as $\psi(2 S)$ [47].

People just note a pair of $\overline{D^{0}}$ and $D^{* 0}$ mesons have a mass threshold $3871.68 \pm 0.08 \mathrm{MeV} / c^{2}$ [7], which is quite close to the $X(3872)$ mass. Thus, it is proposed that $X(3872)$ might be a loosely bound state of two mesons $\left(\bar{D}^{0}\right.$ and $\left.D^{* 0}\right)$ by nuclear force, like a deuteron, which is usually referred to as hadronic molecule state [48]. The molecule interpretation explains lots of decay behavior of $X(3872)$, such as large decay rate to $\overline{D^{0}} D^{* 0}$ [49], Iso-spin violation, etc. However, it suffers from difficulty for the high production rate of $X(3872)$ in high-energy proton anti-proton collision experiment [50]. It finally comes out $X(3872)$ might be a mixture of a charmonium core (5\%) plus big molecule component $(95 \%)$ in its wave function.

Another interpretation is that $X(3872)$ might be a compact object consisting of four quarks (the so-called tetraquark state). Different from the molecule state which is composed of two conventional mesons, tetraquark state is more like a genuine hadron with four quarks tightly confined in a "bag." In order to calculate the tetraquark mass, this model groups two quarks together as a diquark (or diantidiquark) system, which shares similar behavior (such as the "color" freedom) as a single quark in QCD. The tetraquark picture also partly explains the $X$ (3872) feature, such as Iso-spin violation etc. By using the $X(3872)$ mass as input, several partner particles of the $X(3872)$ are predicted from the tetraquark model [51]. Yet, these partners are still not established in the experiment.

\subsection{The vector $Y$-family}

In 2005, the BABAR experiment in US observed a new resonance in $e^{+} e^{-} \rightarrow \pi^{+} \pi^{-} J / \psi$ process with mass near $4.26 \mathrm{GeV} / c^{2}$, which was named as $Y(4260)$ [19]. To produce the resonance, the experiment use an initial-stateradiation method, which yields the spin-parity quantum numbers $J^{P C}=1^{--}$for the $Y(4260)$ resonance. The $Y$ (4260) signal is later confirmed by the Belle [26] and CLEO-c [52] experiments. The $Y(4260)$ mass is well above a pair of charm mesons $\left(D \bar{D}, D \bar{D}^{*}\right.$, and $\left.D^{*} \bar{D}^{*}\right)$. According to the potential model, vector charmonium state with mass above the open-charm threshold should dominantly decay to charm meson pairs. However, by far, we never see the decay of $Y(4260)$ to open charm mesons. Instead, the $Y(4260)$ tends to have large decay width to hidden charm $U / \psi$ and $\psi(2 S)]$ final states. And there is also no position for the $Y(4260)$ in the charmonium spectrum (c.f. Fig. 2).

A recent precise measurement of $e^{+} e^{-} \rightarrow \pi^{+} \pi^{-} J / \psi$ cross section at BESIII in fact finds that the previous $Y(4260)$ is an overlap of two narrow resonances-the $Y(4220)$ and $Y$ (4320) [53]. In addition, experimentally, we find more vector candidates. In the $e^{+} e^{-} \rightarrow \pi^{+} \pi^{-} \psi(2 S)$ process using the same initial-state-radiation method, the Belle experiment observed the $Y(4360)$ and $Y(4660)$ resonances [20], which are also confirmed by $B A B A R$ [54] and BESIII [55]. At the moment, all the vector charmonium states between 3.77 and $4.5 \mathrm{GeV}$ predicted by the potential model are well established [7]. The appearance of these newly observed vector states obviously makes an overpopulation of the charmonium spectrum 
and hints us that some of them should be exotic states. The $Y(4260)$ once was considered as a candidate for a hybrid state, as the lattice QCD calculate the charmonium hybrid mass around $4285 \mathrm{MeV} / c^{2}$ [56]. However, the precise mass measurement from BESIII experiment challenges this interpretation [53]. Other interpretations, such as hadronic molecule state, are also very popular [48].

\subsection{The charged $Z_{c}$ states}

For quite some time, people are not confident about the exotic hadrons, as they are electric neutral (such as the $X(3872)$ and $Y(4260)$ ) and it is difficult to ruled out the possibility from an un-known (or not well known) charmonium state. With the discovery of charged $Z_{c}$ state, these doubts are eventually gone. The first charged candidate $Z_{c}(4430)$ was reported by the Belle experiment in $B \rightarrow K \pi^{ \pm} \psi(2 S)$ decay [57]; unfortunately, it is not confirmed by the BABAR experiment in the same process [58]. The first convincing charged state is $Z_{c}(3900)$, which is described in Section 3 in details. Until then, there are many more charged $Z_{c}$ states reported from experiments [7], c.f. Table 1. Among them, the $Z_{c}(3900)$ resonance is the most solid one, as it is observed by 4 experiments. Its Iso-spin partner $Z_{c}(3900)^{0}$ is also established in $\pi^{0} \pi^{0} J / \psi$ process by BESIII [59].

The charged feature makes $Z_{c}$ states not possible to be a conventional charmonium state, but exotic hadrons. At the moment, people have no doubt about their exotic nature, but are more interested in their internal structures, i.e., how do multi-quarks bound together to form such a particle? Take the $Z_{c}(3900)$ as an example, there are two popular interpretations. One proposes that $Z_{c}(3900)$ is a hadronic molecule state, built of $D^{ \pm} D^{* 0}$ (or $D^{0} D^{* \pm}$ ) meson pair [48]. The $Z_{c}$ (3900) mass seems to match the $D^{ \pm} D^{* 0} / D^{0} D^{* \pm}$ mass threshold, which is favored by the molecule model. The other proposes $Z_{c}(3900)$ is a tetraquark particle [60], which also agrees with early predictions for its mass. There are also voices that $Z_{c}(3900)$ might be due to kinematic effect [61], but such kind of

Table 1 The charged $Z_{c}$ states reported in experiments

\begin{tabular}{lll}
\hline Charged states & Mass $\left(\mathbf{M e V} / \mathbf{c}^{\mathbf{2}}\right)$ & Observation \\
\hline$Z_{c}(3900)^{ \pm, 0}$ & 3887 & BESIII/Belle/CLEO-c/D0 \\
$Z_{c}(4020)^{ \pm, 0}$ & 4024 & BESIII \\
$Z_{C}(4050)^{ \pm}$ & 4051 & Belle \\
$Z_{c}(4100)^{ \pm}$ & 4096 & LHCb \\
$Z_{c}(4200)^{ \pm}$ & 4196 & Belle \\
$Z_{C}(4240)^{ \pm}$ & 4239 & LHCb \\
$Z_{c}(4250)^{ \pm}$ & 4248 & Belle \\
$Z_{c}(4430)^{ \pm}$ & 4478 & Belle/LHCb \\
\hline
\end{tabular}

explanation is lack of clear physics behind the model. More studies are still needed to reveal the internal structure of four-quark particles.

\subsection{The pentaquark}

In 2015, the LHCb experiment reported observation of two charged states, $P_{c}(4380) / P_{c}(4450) \rightarrow p J / \psi$ in the $\Lambda_{b} \rightarrow K^{-} p J / \psi$ process [62]. The mass and width for $P_{c}(4380)$ are $4380 \pm 8 \pm 29 \mathrm{MeV} / c^{2}$ and $205 \pm 18 \pm 86 \mathrm{MeV}$; for $P_{c}(4450)$ are $4450 \pm 2 \pm 3 \mathrm{MeV} / c^{2}$ and $39 \pm 5 \pm$ $19 \mathrm{MeV}$. The spin-parity $\left(J^{P}\right)$ are determined to be $\frac{3}{2}^{+} / \frac{5}{2}^{-}$ or $\frac{5}{2}^{+} / \frac{3}{2}^{-}$(these two cases are not able to be distinguished due to limited statistics) for $P_{c}(4380) / P_{c}(4450)$. The $P_{c}$ states decay to a proton plus a $J / \psi$. Similar to the $Z_{c}$ states, they are charged and cannot be conventional charmonium state. They should contain five quarks inside-so-called pentaquark. In 2019, the LHCb experiment updates the study with 10 times more statistics. A narrow $P_{c}(4312)$ state is further observed, and the $P_{c}(4450)$ splits into two $-P_{c}(4440)$ and $P_{c}(4457)$ [63], respectively. Regarding the nature of pentaquark, it is similar to charged $Z_{c}$ states. Due to matching some baryon-meson pair mass threshold $\left(\Sigma_{c}^{+} \overline{D^{0}} / \Sigma_{c}^{+} D^{-}\right)$, the hadronic molecule interpretation is popular [48].

\section{Summary}

The traditional Quark Model works well over the past half century. In 2013, both the BESIII experiment and the Belle experiment observed the charged $Z_{c}(3900)$ state, which is the first convincing particle consisted of four quarks. $Z_{c}$ (3900), together with other newly observed $X Y Z$ particles, obviously show the existence of QCD exotic hadrons, which refreshes our knowledge about the hadronic matter in the universe. About the detailed structure of these new particles, it is still in progress at the moment. Theoretically, we propose various models, such as molecule, and tetraquark to explain their nature. Experimentally, more and more data is coming, such as the BESIII experiment in China, the Belle II experiment in Japan, the LHCb experiment in Europe, and the $\bar{P}$ ANDA experiment in Germany, which will give more precise measurements. With the study of $X Y Z$ particles, we are entering into a new era of modern hadron spectroscopy, which will finally deepen our understanding about the QCD theory and also the strong interaction.

\footnotetext{
Acknowledgements

I would like to thank my PhD advisor, Prof. Changzheng Yuan from IHEP, China. We collaborate in research for many years. I also thank my BESIII and Belle collaborators, who operate the experiments successfully. This work is supported by the Thousand Talents Programme for Young Professionals and the National Natural Science Foundation of China (NSFC) under Contracts No. 11975141.

Authors' contributions

The authors read and approved the final manuscript.
} 


\section{Competing interests}

The author declares that he has no competing interests.

Received: 24 December 2020 Accepted: 26 January 2021

Published online: 25 March 2021

\section{References}

1. M. Gell-Mann, California Institute of Technology Synchrotron Laboratory Report CTSL-20 (1961). Phys. Rev. 125, 1067 (1962)

2. Y. Ne'eman. Nucl. Phys. 26, 222 (1961)

3. S. Okubo. Progr. Theoret. Phys. (Kyoto). 27, 949 (1962)

4. V. E. Barnes, et al. Phys. Rev. Lett. 12, 204 (1964)

5. M. Gell-Mann. Phys. Lett. 8, 214 (1964); G. Zweig, CERN Report 8182/TH.401, 1 (1964)

6. R. Aaij, et al. (LHCb Collaboration), Phys. Rev. Lett. 119, 112001 (2017); 121, 052002 (2018)

7. P. A. Zyla, et al. (Particle Data Group), Prog. Theor. Exp. Phys. 2020, $083 C 01$ (2020)

8. H. Chen, et al. Phys. Rep. 639, 1-121 (2016)

9. S. Olsen, T. Skwarnicki, D. Zieminska. Rev. Mod. Phys. 90, 015003 (2018)

10. N. Brambilla, et al. Phys. Rep. 873, 1-154 (2020)

11. W. Lee, D. Weingarten. Phys. Rev. D. 61, 014015 (1999)

12. S. K. Choi, et al, Belle Collaboration. Phys. Rev. Lett. 91, 262001 (2003)

13. E. Eichten, et al. Phys. Rev. D. 17, 3090 (1978); 21, 203 (1980)

14. S. Godfrey, N. Isgur. Phys. Rev. D. 32, 189 (1985)

15. J. J. Aubert, et al. Phys. Rev. Lett. 33, 1404-1406 (1974); J. E. Augustin, et al., Phys. Rev. Lett. 33, 1406-1408 (1974)

16. J. L. Rosner, et al. (CLEO Collaboration), Phys. Rev. Lett. 95, 102003 (2005); P. Rubin, et al. (CLEO Collaboration), Phys. Rev. D. 72, 092004 (2005)

17. M. Ablikim, et al, BESIII Collaboration. Phys. Rev. Lett. 115, 011803 (2015)

18. R. Aaij, et al, LHCb Collaboration. J. High Energy Phys. 07, 035 (2019)

19. B. Aubert, et al, BABAR Collaboration. Phys. Rev. Lett. 95, 142001 (2005)

20. X. L. Wang, et al, Belle Collaboration. Phys. Rev. Lett. 99, 142002 (2007)

21. M. Ablikim, et al, BESIII Collaboration. Phys. Rev. Lett. 110, 252001 (2013)

22. Z. Q. Liu, et al, Belle Collaboration. Phys. Rev. Lett. 110, 252002 (2013)

23. R. A. Briere, et al., (CLEO-c and CESR-c Taskforce, CLEO Collaboration). CLNS 01/1742, 1-270 (2001)

24. M. Ablikim, et al, BESIII Collaboration. Nucl. Instrum. Methods Phys. Res. Sect. A. 614, 345 (2010)

25. E. V. Abakumova, et al. Nucl. Instrum. Meth. A. 659, 21 (2011)

26. C. Z. Yuan, et al, Belle Collaboration. Phys. Rev. Lett. 99, 182004 (2007)

27. M. Ablikim, et al, BESIII Collaboration. Phys. Rev. Lett. 119, 072001 (2017)

28. D. Powell. Nature. 498, 280-281 (2013)

29. Mysterious Subatomic Particle May Represent Exotic New Form of Matter https://www.wired.com/2013/06/four-quark-particle/

30. Mögliche neue Materieform: Physiker entdecken mysteriöses Teilchen. https://www.spiegel.de/thema/higgs_boson/

31. M. Rini, J. Thomas. Physics. 6, 139 (2013)

32. T. Xiao, et al. Phys. Lett. B. 727, 366 (2013)

33. V. M. Abazov, et al, D0 Collaboration. Phys. Rev. D. 100, 012005 (2019)

34. D. Acosta, et al. (CDF Collaboration), Phys. Rev. Lett. 93, 072001 (2004)

35. V. M. Abazov, et al., (D0 Collaboration). Phys. Rev. Lett. 93, 162002 (2004)

36. B. Aubert, et al., (BABAR Collaboration). Phys. Rev. D. 71, 071103 (2005)

37. D. Fasanella, POS EPS-HEP2011. 177 (2011)

38. R. Aaij, et al., (LHCb Collaboration). Eur. Phys. J. C. 72, 1972 (2012)

39. M. Ablikim, et al., (BESIII Collaboration). Phys. Rev. Lett. 112, 092001 (2014)

40. K. Kordas, PoS DIS2016. 160 (2016)

41. R. Aaij, et al, LHCb Collaboration. J High Energy Phys. 08, 123 (2020)

42. R. Aaij, et al, LHCb Collaboration. Phys. Rev. Lett. 110, 222001 (2013)

43. B. Aubert, et al, BABAR Collaboration. Phys. Rev. D. 71, 031501 (2005)

44. S.-K. Choi, et al, The Belle Collaboration. Phys. Rev. D. 84, 052004 (2011)

45. A. Abulencia, et al, CDF Collaboration. Phys. Rev. Lett. 96, 102002 (2006)

46. M. Ablikim, et al, BESIII Collaboration. Phys. Rev. Lett. 122, 202001 (2019)

47. C. Hanhart, et al. Phys. Rev. D. 85, 011501 (2012)

48. F. K. Guo, et al. Rev. Mod. Phys. 90, 015004 (2018)

49. M. Ablikim, et al, BESIII Collaboration. Phys. Rev. Lett. 124, 242001 (2020)

50. C. Bignamini, et al. Phys. Rev. Lett. 103, 162001 (2009)

51. L. Maiani, et al. Phys. Rev. D. 71, 014028 (2005)

52. Q. He, et al, CLEO Collaboration. Phys. Rev. D. 74, 091 104(R) (2006)

53. M. Ablikim, et al, BESIII Collaboration. Phys. Rev. Lett. 118, 092001 (2017)

54. J. P. Lees, et al, BaBar Collaboration. Phys. Rev. D. 89, 111103 (2014)

55. M. Ablikim, et al, BESIII Collaboration. Phys. Rev. D. 96, 032004 (2017)

56. L. Liu, et al, Hadron Spectrum collaboration. J. High Energy Phys. 07, 126 (2012)
57. S.-K. Choi, et al, Belle Collaboration. Phys. Rev. Lett. 100, 142001 (2008)

58. B. Aubert, et al, BABAR Collaboration. Phys. Rev. D. 79, 112001 (2009)

59. M. Ablikim, et al, BESIII Collaboration. Phys. Rev. Lett. 115, 112003 (2015)

60. L. Maiani, et al. Phys. Rev. D. 87, 111102(R) (2013)

61. D. Y. Chen, X. Liu, T. Matsuki. Phys. Rev. D. 88, 036008 (2013)

62. R. Aaij, et al, LHCb Collaboration. Phys. Rev. Lett. 115, 072001 (2015)

63. R. Aaij, et al, LHCb Collaboration. Phys. Rev. Lett. 122, 222001 (2019)

\section{Publisher's Note}

Springer Nature remains neutral with regard to jurisdictional claims in published maps and institutional affiliations.

\section{Submit your manuscript to a SpringerOpen ${ }^{\circ}$ journal and benefit from:}

- Convenient online submission

- Rigorous peer review

- Open access: articles freely available online

- High visibility within the field

- Retaining the copyright to your article

Submit your next manuscript at $>$ springeropen.com 